\title{
2465. Optimal design of the main support structure of space camera aiming at the RMS value of random response
}

\author{
Lin Li ${ }^{1}$, Luyang Tan ${ }^{2}$, Dong Wang ${ }^{3}$, Hongbo Yang ${ }^{4}$ \\ $1,2,3,{ }^{4}$ Changchun Institute of Optics, Fine Mechanics and Physics, Chinese Academy of Sciences, \\ Changchun, China \\ ${ }^{1,2}$ University of Chinese Academy of Sciences, Beijing, China \\ ${ }^{3}$ Chang Guang Satellite Technology Ltd, Changchun, China \\ ${ }^{3}$ Corresponding author

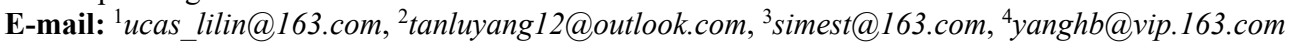

Received 4 August 2016; received in revised form 26 December 2016; accepted 9 January 2017

DOI https://doi.org/10.21595/jve.2017.17516

Check for updates

\begin{abstract}
To explore the optimal design method for main support structure of micro satellite, this paper proposed a method targeting the random acceleration response RMS value of the space camera installation position when design the main support structure of LQ-video satellite in Jilin-1 group satellites. Camera main support structure optimization mathematical model was established, and the thickness and flexible beam position of the flexible beam support structure has been optimized in the establishment of the optimization mathematical model. When the flexible beam thickness is $2.5 \mathrm{~mm}$, and the distance between it and the support structure mounting surface is $94.5 \mathrm{~mm}$, the camera installation point acceleration response root mean square (RMS) value is minimal. Engineering analysis showed that the maximal random response RMS of the camera installation point is 19.6 grms and the maximal relative magnification is 0.93 . The camera mechanics test showed that the maximal relative error of finite element analysis and experimental measurements is $4.0 \%$ and the maximal relative magnification of the response is 1.2 which is less than the overall index 1.5. It proved that the optimization method is effective and feasible.
\end{abstract}

Keywords: micro satellite, random vibration, space camera, main support structure, optimal design.

\section{Nomenclature}

M n-order mass matrix

C Damping matrix

K Stiffness matrix

X Displacement vector

$\dot{X} \quad$ Velocity vector

$\ddot{\mathbf{X}} \quad$ Acceleration vector

$f(t) \quad$ System incentives

$\ddot{Y}(t) \quad$ The acceleration incentive

$S_{\ddot{Y}}(w)$ Power spectral density of acceleration incentive

E Acceleration vector

$w_{i} \quad$ System $n$-degree natural frequency

$x_{i} \quad$ Mode of vibration

$\phi_{i} \quad$ Mass normalized vibration mode

$\xi_{i} \quad$ System $i$ th modal damping ratio

$\Upsilon_{i} \quad i$ th mode participation coefficient

$u_{i} \quad i$ th modal displacement

$h_{i}(\tau) \quad i$ th impulse response function

$\ddot{R}_{Y} \quad$ Input auto correlation function

$H_{i}(w)$ Frequency transfer function

$h_{i}(t)$ Impulse response function 


$\begin{array}{ll}\lambda_{i} & \text { ith isolated eigen values } \\ \left\{\varphi_{i}\right\} & \text { Mode vectors } \\ \lambda_{i, j} & \text { Derivative of } \lambda_{i} \text { under the design variable } t_{j}(j=1,2, \ldots, L) \\ \left\{\varphi_{i, j}\right\} & \text { Derivative }\left\{\varphi_{i}\right\} \text { under the design variable } t_{j}(j=1,2, \ldots, L) \\ {\left[K_{, j}\right]} & \text { Derivative matrix of }[K] \text { under the design variable } t_{j} \\ {\left[M_{, j}\right]} & \text { Derivative matrix of }[M] \text { under the design variable } t_{j} \\ t_{e} & \text { Thickness of flexible joint } \\ h_{e} & \text { Distance from flexible beam to support structure mounting surface } \\ t_{i} & \text { Thickness variable } \\ h_{i} & \text { Distance variable }\end{array}$

\section{Introduction}

Modern micro-satellite has a strong practicability for small size, light weight, low cost, short development cycle and high performance characteristics [1, 2]. Therefore, the micro satellite has been paid more attention in the field of international space technology. With the vigorous development of micro satellite, the effective load ratio is increasing, and the requirements of the main structure of the satellite are more severe [3-5]. Exploring optimization method is even more urgent for main structure design of micro satellite.

LQ-video satellite in Jilin-1 group satellites is a micro video imaging satellite whose mass is no more than $95 \mathrm{Kg}$. Aiming at reducing the satellite structure mass and volume, it uses space-borne integration design to improve the structure density of micro satellite. The camera is connected with the satellite through the camera main support structure. When the rocket launches, the satellite suffering random acceleration vibration excitation which is integrated by pulsation generation rocket thrust, jet noise and turbulent boundary layer noise [6-8]. If the acceleration vibration directly passed through the main support structure to the camera, it will cause the camera structural deformation or even destroyed. Therefore, the camera main support structure should be optimized to gain a better dynamic performance under a given frequency band.

The existing research, based on random vibration theory and test, mostly examined the structural strength of the satellite rather than optimized the structure of the dynamic parameters [9-12]. For instances, W. Jung, A. P. Mazzoleni, J. Chung [13] presents a dynamic analysis of a tethered satellite system with a moving mass. The analysis results show that a one-piece dumbbell model is qualitatively valid for studying the system under certain conditions, such as when the initial liberation angles, moving mass velocity, and moving mass size are small, the tether length is large, and the mass ratio of the two satellites is large. You B., Zhang H., Li W., et al. [14] presented a methodology for modeling and analysis of satellite antenna systems considering the effects of the joint clearance and reflector flexibility in the system. Based on a thorough geometric description of the eccentricity vector, the multibody dynamics of satellite antenna are analyzed by considering a flexible reflector with ideal and nonideal joints.

According to LQ-video satellite overall design requirements, this paper proposed a method to optimize design camera main support structure targeting the random vibration response RMS value of the camera installation position, and established a random vibration response optimization model. The thickness of the flexible joint and flexible beam position has been optimized. In order to verify the optimization results, engineering analysis and mechanical testing of the optimized camera main support structure are carried out. The results showed that the optimization designed main support structure match the overall satellite design specifications well, and the optimize design method is effective and feasible.

\section{Optimization model}

Size optimization is one of the most important topology optimization method, especially used 
in structure optimization [15-17]. It refers to a mathematical method for optimizing material distribution in a given area under a given load conditions, constraints and performance indicators. The optimization mathematical is established from the objective function, design variables and constraint conditions, and the optimization process is explained in this section.

\subsection{Systematic random response function}

Spectrum analysis method is generally used in the random response. An $\mathrm{n}$ degrees' freedom linear system is expressed by random excitation and its kinetic equation $[18,19]$ is represented as Eq. (1):

$$
\mathbf{M} \ddot{\mathbf{X}}+\mathbf{C} \dot{\mathbf{X}}+\mathbf{K X}=f(t),
$$

where, $\mathbf{M}, \mathbf{C}$ and $\mathbf{K}$ respectively represent $n$-order mass matrix, damping matrix and stiffness matrix. $\mathbf{X}$ is displacement vector, $\dot{\mathbf{X}}$ is velocity vector, $\ddot{\mathbf{X}}$ is acceleration vector and $f(t)$ is system incentives.

Assume the acceleration incentive is $\ddot{Y}(t)$, and its power spectral density (PSD) is $S_{\ddot{Y}}(w)$, the kinetic random vibration equation is represented as Eq. (2):

$$
\mathbf{M} \ddot{\mathbf{X}}+\mathbf{C} \dot{\mathbf{X}}+\mathbf{K X}=\mathbf{M E} \ddot{Y}(t),
$$

where, $\mathbf{E}$ indicates an acceleration vector.

First, to solve system's intrinsic mode, it should be $C=0, f(t)=0$ and the system free motion equation [20] is represented as Eq. (3):

$\mathbf{M X}+\mathbf{K X}=0$.

The corresponding characteristic equation is represented as Eq. (4):

$\mathbf{K}-w^{2} \mathbf{M}=0$.

System $n$-degree natural frequency is $w_{i}$, and the corresponding mode of vibration is $x_{i}, i=1,2, \ldots, n$. where, $m_{i}=x_{i}^{T} \mathbf{M} x_{i}, k_{i}=x_{i}^{T} k x_{i}, w_{i}^{2}=k_{i} / m_{i}$, the mode of vibration $x_{i}$ multiplies a constant multiplier and the mass normalized vibration mode is $\phi_{i}$. In classical damping, the Eq. (2) rewrite as follows:

$\ddot{u}_{i}+2 w_{i} \xi_{i} \dot{u}_{i}+w_{i}^{2} u_{i}=\Upsilon_{i} \ddot{Y}(t), \quad(i=1,2, \ldots, n)$.

In this formula, $\xi_{i}$ is the system $i$-th modal damping ratio; $\Upsilon_{i}$ is the $i$-th mode participation coefficient:

$\Upsilon_{i}=\phi^{T} \mathbf{M E}$

Relationship between $\mathbf{X}(t)$ and $u_{i}$ is:

$\mathbf{X}(t)=\left[\begin{array}{llll}\phi_{1} & \phi_{2} & \cdots & \phi_{n}\end{array}\right]\left[\begin{array}{c}u_{1} \\ u_{2} \\ \vdots \\ u_{1}\end{array}\right]=\Phi u(t)=\sum_{i=1}^{n} u_{i} \phi_{i}$.

Solution of Eq. (5) in the time domains is: 
$u_{i}=\Upsilon_{i} \int_{-\infty}^{+\infty} h_{i}(\tau) \ddot{Y}(t-\tau) d \tau$

where, $h_{i}(\tau)$ is the $i$-th impulse response function.

Through Eqs. (7)-(8), we get:

$X(t)=\sum_{i=1}^{n} \Upsilon_{i} \phi_{i} \int_{-\infty}^{+\infty} h_{i}(\tau) \ddot{Y}(t-\tau) d \tau$

The auto correlation function of the matrix $\mathbf{X}(t)$ is:

$$
\begin{aligned}
R_{X X} & (\tau)=\mathbf{E}\left[\mathbf{X}(\tau) \mathbf{X}^{T}(t+\tau)\right] \\
= & \sum_{i=1}^{n} \sum_{j=1}^{n} \Upsilon_{i} \Upsilon_{j} \phi_{i} \phi_{i}^{T} \cdot \int_{-\infty}^{+\infty} \int_{-\infty}^{+\infty} \mathbf{E}\left[\ddot{Y}\left(t-\tau_{1}\right) \ddot{Y}\left(t-\tau_{2}+\tau\right)\right] h\left(\tau_{1}\right) h\left(\tau_{2}\right) d \tau_{1} d \tau_{2} \\
= & \sum_{i=1}^{n} \sum_{j=1}^{n} \Upsilon_{i} \Upsilon_{j} \phi_{i} \phi_{i}^{T} \cdot \int_{-\infty}^{+\infty} \int_{-\infty}^{+\infty} \ddot{R}_{Y}\left(\tau+\tau_{1}-\tau_{2}\right) h\left(\tau_{1}\right) h\left(\tau_{2}\right) d \tau_{1} d \tau_{2} .
\end{aligned}
$$

According to the Wiener-Khintchine relationship [21], output PSD is the Fourier transform of the output of autocorrelation function. By exchanging integral order, and introducing variable $\theta=t+\tau_{1}-\tau_{2}$, we get output PSD function as follows:

$S_{X X}(w)=\sum_{i=1}^{n} \sum_{j=1}^{n} \Upsilon_{i} \Upsilon_{j} \phi_{i} \phi_{i}^{T} h_{i}\left(\tau_{1}\right) e^{i w \tau_{1}} h_{j}\left(\tau_{2}\right) e^{-i w \tau_{2}} \cdot\left[\frac{1}{2 \pi} \int_{-\infty}^{+\infty} \ddot{R}_{Y}(\theta) e^{-i w \theta} d \theta\right] d \tau_{1} d \tau_{2}$.

Input PSD function $\ddot{S}_{Y}$ is the $\ddot{S}_{Y}$ Fourier transform of input auto correlation function $\ddot{R}_{Y}$, and frequency transfer function $H_{i}(w)$ is the Fourier transform of impulse response function $h_{i}(t)$, then Eq. (11) rewrite as Eq. (12):

$S_{X X}(w)=\sum_{i=1}^{n} \sum_{j=1}^{n} \Upsilon_{i} \Upsilon_{j} \phi_{i} \phi_{i}^{T} H_{i}(w) H_{j}(w) \ddot{S}_{Y}(w)$

Generally, ignoring the cross-order terms to simplify approximation method is used in engineering in Eq. (12), the response PSD abbreviated as follows:

$S_{X X}(w)=\sum_{i=1}^{n} \Upsilon_{i}^{2} \phi_{i} \phi_{i}^{T}\left|H_{i}(w)\right|^{2} \ddot{S}_{Y}(w)$.

The random acceleration RMS response expression which is also the optimization objective function shown in Eq. (14):

$\sigma_{X X}^{2}=\int_{-\infty}^{+\infty} S_{X X}(w) d w=\sum_{i=1}^{n} \Upsilon_{i}^{2} \phi_{i} \phi_{i}^{T} \int_{-\infty}^{+\infty}\left|H_{i}(w)\right|^{2} \ddot{S}_{Y}(w) d w$.

\subsection{System sensitivity}

Modal analysis is used to determine the vibration characteristics of the structure. It is mainly about the natural frequency and vibration mode of the system structure, which is an important 
index to investigate the dynamic stiffness. Structural low order mode is also an important basis for modifying the structure. The modal sensitivity of the structure is analyzed as follows.

$\lambda_{i}$ and $\left\{\varphi_{i}\right\}$ are the $i$-th isolated eigen values and the corresponding mode vectors, which satisfy the characteristic Eq. (15):

$\left([\mathbf{K}]-\lambda_{i}[\mathbf{M}]\left\{\varphi_{i}\right\}=0\right.$.

$\lambda_{i, j}$ and $\left\{\varphi_{i, j}\right\}$ are the derivative of $\lambda_{i}$ and $\left\{\varphi_{i}\right\}$ under the design variable $t_{j}(j=1,2, \ldots, L)$, $\left[\mathbf{K}_{, j}\right]$ and $\left[\mathbf{M}_{, j}\right]$ are the derivative matrix of $[\mathbf{K}]$ and $[\mathbf{M}]$ under the design variable $t_{j}$. Eigen values sensitivity $\lambda_{i, j}$ and modal vector sensitivity $\left\{\varphi_{i, j}\right\}$ are as follow:

$\lambda_{i, j}=\left\{\varphi_{i}\right\}^{T}\left(\left[\mathbf{K}_{, j}\right]-\lambda_{i}\left[\mathbf{M}_{, j}\right]\right)\left\{\varphi_{i}\right\}\left\{\varphi_{i, j}\right\}$,
$\left\{\varphi_{i, j}\right\}=\sum_{\substack{k=1 \\ k \neq i}}^{n} \frac{1}{\lambda_{i}-\lambda_{k}}\left(\left\{\varphi_{k}\right\}^{T}\left(\left[\mathbf{K}_{, j}\right]-\lambda_{i}\left[\mathbf{M}_{, j}\right]\right) \cdot\left\{\varphi_{i}\right\}\right)-\frac{1}{2}\left(\left\{\varphi_{i}\right\}^{T}\left[\mathbf{M}_{, j}\right]\left\{\varphi_{i}\right\}\right)\left\{\varphi_{i}\right\}$.

To calculate $\lambda_{i, j}$ and $\left\{\varphi_{i, j}\right\}$, it should be calculate $\left[\mathbf{K}_{, j}\right]=\frac{\partial[\mathbf{K}]}{\partial t_{j}}=\sum \frac{\partial[\mathbf{K}]^{e}}{\partial t_{j}}$ and $\left[\mathbf{M}_{, j}\right]=\frac{\partial[\mathbf{M}]}{\partial t_{j}}=\sum \frac{\partial[\mathbf{M}]^{e}}{\partial t_{j}}$ first. In this paper, shell thickness $t$ is used in the optimization analysis. Therefore, the derivative matrix of the shell element matrix $C$ and the derivative matrix of the mass matrix $D$ are given.

$$
\begin{gathered}
\frac{\partial[\mathbf{K}]^{e}}{\partial t_{j}}=\frac{[\mathbf{K}]^{e}}{t_{j}}, \\
\frac{\partial[\mathbf{M}]^{e}}{\partial t_{j}}=\frac{[\mathbf{M}]^{e}}{t_{j}} .
\end{gathered}
$$

Derivative Eq. (14), we would get random acceleration response sensitivity.

\subsection{Design variables}

Support structure flexible beam thickness $T$ and distance from flexible beam to support structure mounting surface $H$ are written as Eq. (20):

$\mathbf{T}^{\prime}=\left(t_{1}, t_{2}, t_{3}, \ldots t_{e} \ldots t_{n}\right)^{T}, \quad \mathbf{H}^{\prime}=\left(h_{1}, h_{2}, h_{3}, \ldots h_{e} \ldots h_{n}\right)^{T}$,

where, $t_{e}$ is thickness of flexible joint, $h_{e}$ is distance from flexible beam to support structure mounting surface.

\subsection{Mathematical model}

LQ-video satellite requires that the primary frequency of the camera main support structure is greater than the camera primary frequency $110 \mathrm{~Hz}$. The safety factor is 1.2 , the primary frequency of the camera main support structure is no less than $132 \mathrm{~Hz}$. The $t_{e}$ and $h_{e}$ satisfy the Eq. (21):

$1 \leq t_{i} \leq t_{e} \leq 5$,

$6.5 \leq h_{i} \leq h_{e} \leq 105.5$,

where, $t_{i}$ represents the thickness variable, $h_{i}$ represents the distance variable.

The camera main support structure size optimization mathematical model is expressed as Eq. (22): 
find: $\mathbf{T}^{\prime}=\left(t_{1}, t_{2}, \cdots, t_{e}, \cdots t_{n}\right)^{T}$,

$$
\mathbf{H}^{\prime}=\left(h_{1}, h_{2}, h_{3}, \ldots h_{e} \ldots h_{n}\right)^{T},
$$

Minimize: $C(x)=\sigma_{x x}{ }^{2}$,

Subject to: $f_{1} \geq 132$,

$$
\begin{aligned}
& 1 \leq t_{i} \leq t_{e} \leq 5, \\
& 6.5 \leq h_{i} \leq h_{e} \leq 105.5 .
\end{aligned}
$$

Until now, the random vibration mean square response is converted into a deterministic harmonic response analysis problem based on the virtual excitation method, and the sensitivity of the modal sensitivity and random acceleration response are analyzed.

\section{Optimization process and results}

According to the mathematical model in Section 2, the sizing optimization was introduced to optimize the thickness of the flex joint and the flex joint position in the main support structure of the camera. The optimization design flow is shown in Fig. 1.

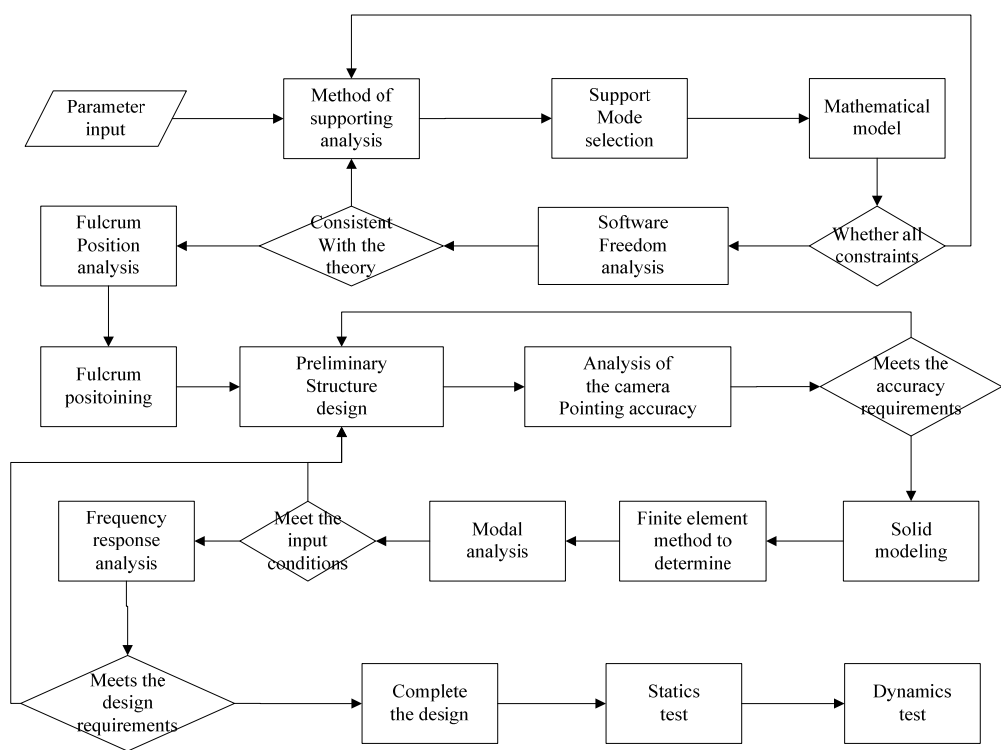

Fig. 1. Flow chart

Table 1. The acceleration power spectrum (PSD)

\begin{tabular}{|c|c|}
\hline Frequency $(\mathrm{Hz})$ & Power spectral density \\
\hline $20-150$ & $+3 \mathrm{~dB} / \mathrm{oct}$ \\
\hline $150-280$ & $0.08 \mathrm{~g}^{2} / \mathrm{Hz}$ \\
\hline $280-320$ & $0.30 \mathrm{~g}^{2} / \mathrm{Hz}$ \\
\hline $320-380$ & $0.20 \mathrm{~g}^{2} / \mathrm{Hz}$ \\
\hline $380-850$ & $0.10 \mathrm{~g}^{2} / \mathrm{Hz}$ \\
\hline $850-1000$ & $0.04 \mathrm{~g}^{2} / \mathrm{Hz}$ \\
\hline $1000-2000$ & $0.01 \mathrm{~g}^{2} / \mathrm{Hz}$ \\
\hline
\end{tabular}

Overall design of the LQ-video satellite requires that the height of main support structure of the camera is $118 \mathrm{~mm}$. The material of the main supporting structure is Titanium alloy TC4 which is provided with low density and high strength.

The applied loads are the random acceleration which is from the rocket in launching. The acceleration power spectrum is shown in Table 1, and the RMS value of PSD is $10.16 \mathrm{~g}$. Structural 
damping is 0.03 in this model.

Optimize step size is $0.5 \mathrm{~mm}$ during the optimization design process. After 69 steps of iteration, the random vibration acceleration response of the camera mounting point is minimal and thickness of flexible joint $t_{e}$ is $2.5 \mathrm{~mm}$, distance $h_{e}$ is $94.5 \mathrm{~mm}$. Optimization iterative process convergence curve is shown in Fig. 2.

\section{Finite element numerical analysis}

To estimate the optimization result, the finite element analysis is carried out by using MSC. Patran\&Nastran 2013. FEM includes secondary mirror (SM), SM linker, SM substrate, SM light shield, SM support, Primary mirror (PM), PM substrate, base plate and camera support structure which contains 56614 nodes, 35532 elements, 12 RBE2 and 1 CONM2. FEM is shown in Fig. 3.

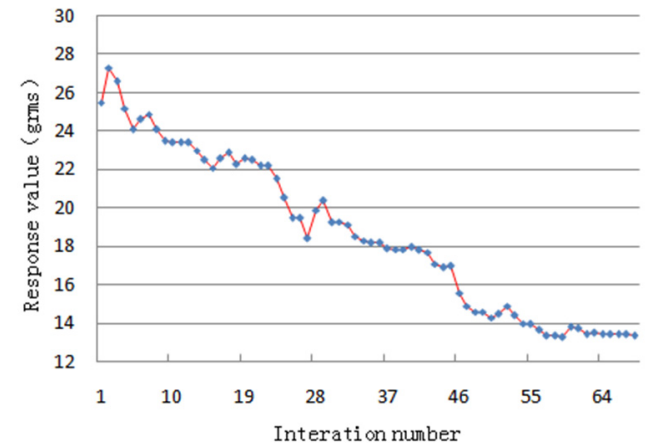

Fig. 2. Iterative convergence curve

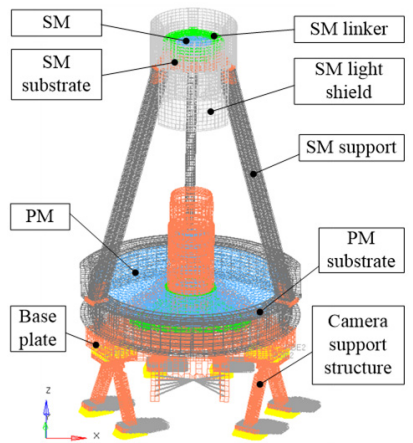

Fig. 3. Finite element model

Table 2. Main materials parameters

\begin{tabular}{|c|c|c|c|c|}
\hline Name & Material & Elastic modulus (MPa) & $\mathrm{Nu}$ & Density $\left(\mathrm{kg} / \mathrm{m}^{3}\right)$ \\
\hline SM & \multirow{2}{*}{ Zerodur } & \multirow{2}{*}{91} & \multirow{2}{*}{0.24} & \multirow{2}{*}{2500} \\
\hline $\mathrm{PM}$ & & & & \\
\hline SM linker & \multirow{5}{*}{$\begin{array}{l}\text { Titanium alloy } \\
\text { (TC4) }\end{array}$} & \multirow{5}{*}{110} & \multirow{5}{*}{0.34} & \multirow{5}{*}{4400} \\
\hline SM support & & & & \\
\hline SM substrate & & & & \\
\hline Base plate & & & & \\
\hline $\begin{array}{c}\text { Camera support } \\
\text { structure }\end{array}$ & & & & \\
\hline SM light shield & M40 & 70 & 0.3 & 1500 \\
\hline PM substrate & $\mathrm{T} 700$ & 50 & 0.28 & 1800 \\
\hline
\end{tabular}

Table 3. FE analysis results

\begin{tabular}{|c|c|c|}
\hline Order & Frequency $(\mathrm{Hz})$ & Modal formation \\
\hline 1 & 141.8 & Support structure swing along $X$ axis \\
\hline 2 & 156.4 & Support structure swing along $Y$ axis \\
\hline 3 & 320.7 & Support structure rotation around the $Z$ axis \\
\hline Direction & Input RMS (g) & Output response RMS of sample point (g) \\
\hline$X$-axis & \multirow{3}{*}{10.16} & 15.2 \\
\hline$Y$-axis & & 19.8 \\
\hline$Z$-axis & & 23.3 \\
\hline
\end{tabular}

\section{Experiments}

To verify the feasibility of the size optimization method and the accuracy of the finite element numerical analysis results, we used the model vibration test bench (\# MPA3436/H1859A) to test 
the camera at room temperature according to conditions in Table 1. Test scene is shown in Fig. 4.

Curves of input, analysis and test of the random vibration response of the camera main support structure in the $X$-axis, $Y$-axis and $Z$-axis are shown in Fig. 5. The comparison of random vibration response test results and the analytical data are listed in Table 4.

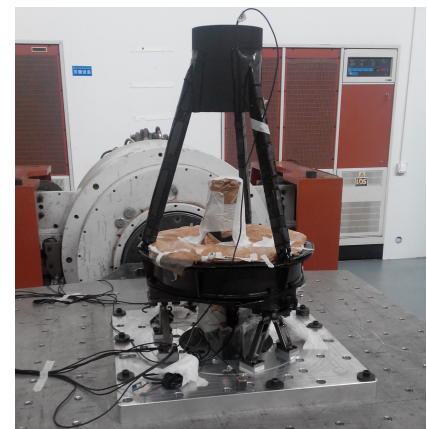

Fig. 4. Placement of random vibration test

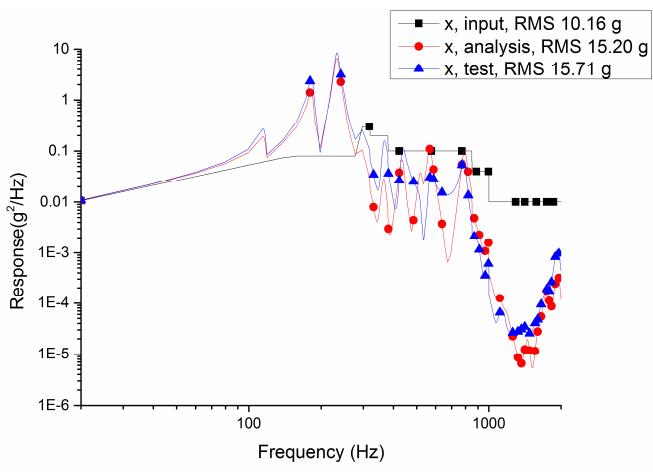

a) $x$ axis

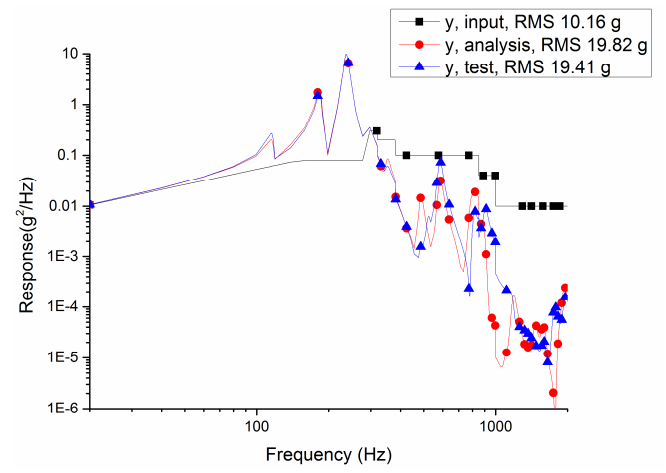

b) $y$ axis

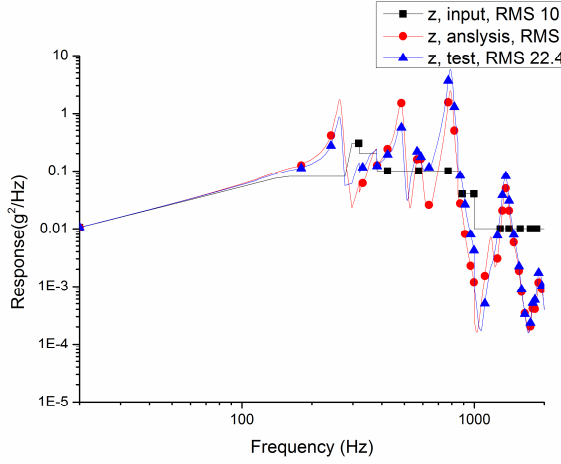

c) $Z$ axis

Fig. 5. Curves of input, analysis and test

Table 4. Compare data of tests and analysis (grms)

\begin{tabular}{|c|c|c|c|c|c|}
\hline Axis & Input & Analysis result & Test result & Relative errors & Relative magnification \\
\hline$x$ & 10.16 & 15.2 & 15.7 & $3.8 \%$ & 0.54 \\
\hline$y$ & 10.16 & 19.8 & 19.4 & $2.1 \%$ & 0.91 \\
\hline$z$ & 10.16 & 23.3 & 22.4 & $4.0 \%$ & 1.2 \\
\hline
\end{tabular}

According to Table 4, the relative errors of random vibration response between finite element analysis results and vibration test data are less than $5 \%$, and the maximum relative magnification 
is 1.2 which is less than overall index 1.5. The results validate correctness of finite element models and feasibility of optimization methods in this paper.

\section{Conclusions}

Traditional optimization methods are mostly based on static mechanical properties parameters or frequency characteristics, which cannot directly optimize the dynamic parameters of the structure. In this paper, due to the RMS value of the acceleration response of the main support structure of the LQ-video satellite in Jilin-1 group satellites is too large under the random vibration excitation, the optimization design method based on the RMS value of the random response is proposed, and the following conclusions are got.

1) The mathematical model of random vibration response optimization is established, the modal and acceleration response sensitivity analysis of the model are carried out, and the optimal design of the main support structure is also provided. This method can provide a new idea for the design of other small satellite main structure.

2) The thickness of the main support structure of the camera is $2.5 \mathrm{~mm}$ and the distance between the support structure and mounting surface is $94.5 \mathrm{~mm}$ after size optimization, the random vibration acceleration response of the camera mounting position is minimum.

3) Engineering analysis and test results show that the main support structure of the camera is $141.8 \mathrm{~Hz}$, which is larger than the overall index $110 \mathrm{~Hz}$, and the maximal relative amplification rate of the support structure is 1.2, which is less than the overall design index 1.5. The main support structure of the camera performance parameters match the overall index well.

\section{Acknowledgements}

The authors would like to thank the financial support of the National Natural Science Foundation for Young Scholar of China (No. 61505203) and Youth Innovation Promotion Association CAS (No. 2011171).

\section{References}

[1] Yatsu Y., Hayashi M., Kawakami K., et al. Development of a micro-satellite TSUBAME for X-ray polarimetry of GRBs. Proceedings of the International Astronomical Union, Vol. 7, Issue 279, 2015, p. 423-424.

[2] Underwood C., Pellegrino S., Lappas V. J., et al. Using cubesat/micro-satellite technology to demonstrate the autonomous assembly of a reconfigurable space telescope (AAReST). Acta Astronautica, Vol. 114, 2015, p. 112-122.

[3] Triharjanto R. H., Poetro R. E., Hardhienata S. Multi-objectives optimization of earth observation micro-satellite design using particle swarm. IEEE International Conference on Aerospace Electronics and Remote Sensing Technology, 2014, p. 192-196.

[4] Safarabadi M., Bazargan S. Prediction of equivalent static loads act on a micro satellite via modal analysis. Engineering Solid Mechanics, Vol. 3, Issue 2, 2015, p. 75-84.

[5] Romero S., Smitz J. Integrated design technique of satellite platform and payload based on microvibration isolators. Spacecraft Recovery and Remote Sensing, Vol. 137, Issue 6, 2014, p. 997-1005.

[6] Dziopa Z., Koruba Z. The impact of launcher turret vibrations control on the rocket launch. Bulletin of the Polish Academy of Sciences Technical Sciences, Vol. 63, Issue 3, 2015, p. 717-728.

[7] Bertin J., Batson J. L. Comparison of cold-gas simulations and rocket-launch data for constrictive launchers. Journal of Spacecraft and Rockets, Vol. 13, Issue 11, 2015, p. 684-691.

[8] Thirty Meter Telescope Detailed Science Case: 2015 Warren Skidmore on behalf of the TMT International Science Development Teams and TMT Science Advisory Committee. Research in Astronomy and Astrophysics, Vol. 12, 2015, p. 1945-2140.

[9] Xiong H., Kong X., Yang Zh., Liu Y. Response regimes of narrow-band stochastic excited linear oscillator coupled to nonlinear energy sink. Chinese Journal of Aeronautics, Vol. 2, 2015, p. 457-468.

[10] Bendsoe M. P., Sigmund O. Topology Optimization: Theory, Methods and Applications. Berlin, Springer, 2003, p. 9-69. 
[11] Lu S. An overview of a unified theory of dynamics of vehicle-pavement interaction under moving and stochastic load. Journal of Modern Transportation, Vol. 3, 2013, p. 135-162.

[12] Tang Ch., Zhang Y., Zhao G., Ma Y. Annoyance rate evaluation method on ride comfort of vehicle suspension system. Chinese Journal of Mechanical Engineering, Vol. 2, 2014, p. 296-303.

[13] Jung W., Mazzoleni A. P., Chung J. Dynamic analysis of a tethered satellite system with a moving mass. Nonlinear Dynamics, Vol. 75, Issues 1-2, 2014, p. 267-281.

[14] You B., Zhang H., Li W., et al. Dynamic analysis of satellite antenna system with joint clearance and reflector flexibility. Journal of Aerospace Engineering, Vol. 27, Issue 2, 2014, p. 297-307.

[15] Bendsoe M. P., Kijuchi N. Generating optical topologies in structural design using a homogenization method. Computer Methods in Applied Mechanics and Engineering, Vol. 71, Issue 2, 1988, p. 193-202.

[16] Cho J. G., Koo J. S., Jung H. S. A lightweight design approach for an EMU carbody using a material selection method and size optimization. Journal of Mechanical Science and Technology, Vol. 30, Issue 2, 2016, p. 673-681.

[17] Tomsia P., Duhovnik J. Simultaneous topology and size optimization of 2D and 3D trusses using evolutionary structural optimization with regard to commonly used topologies. Advances in Mechanical Engineering, Vol. 2014, Issue 1, 2014, p. 1-13.

[18] Qiu Zh, Qiu H. A direct-variance-analysis method for generalized stochastic eigenvalue problem based on matrix perturbation theory. Science China (Technological Sciences), Vol. 6, 2014, p. 1238-1248.

[19] Mischler S., Mouhot C. Exponential stability of slowly decaying solutions to the kinetic-fokkerplanck equation. Archive for Rational Mechanics and Analysis, Vol. 21, Issue 2, 2016, p. 1836-1841.

[20] Yan L., Yang Z., Wang Y. Sine on random vibration based on combined vibration test system. Journal of Vibration and Shock, Vol. 32, Issue 2, 2015, p. 91-95.

[21] Chen Zh, Zhu H., Kuang G. Experimental study on near-field acoustic holography based on the second-order cyclic statistics. Journal of Vibration and Shock, Vol. 30, Issue 9, 2011, p. 202-206.
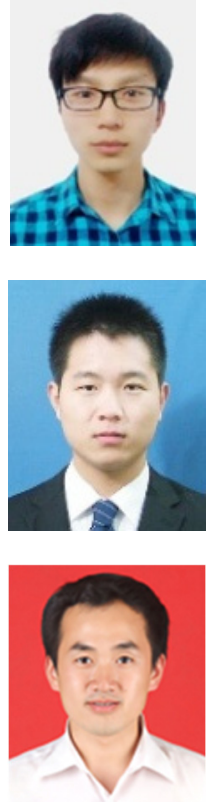

Dong Wang is a researcher and Ph.D. supervisor at University of Chinese Academy of Sciences. He received the Ph.D. degree from the University of Chinese Academy of Science in 2008. His current research interests are satellite overall design and spacecraft thermal control technology.

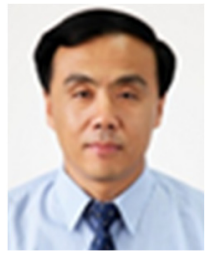

Hongbo Yang is a researcher and Ph.D. supervisor at University of Chinese Academy of Sciences. He is also a Ph.D. supervisor at University of Science and Technology of China. His current research interests are development and application of integrated simulation technology for opto-mechanical and thermal, CAE, opto-mechanical and electrical integration technology. 